\title{
COMPARISON OF MARGINAL AND INTERNAL FIT OF COPINGS FABRICATED FROM POLYETHERETHERKETONE AND ZIRCONIA: AN IN-VITRO STUDY
}

\author{
Mohammad Ramadan Makky *, Tamer Elhamy Shokry **, Mohammad Fawzy Metwally ***
}

\begin{abstract}
Objective: This study evaluated the marginal and internal fit of pressable versus machinable polyetheretherketone (PEEK) and versus zirconia copings. Material and Methods: Twenty one copings of lower molar were used in this study, which were divided according to type of material used and method of manufacturing into three main groups: seven specimens each, zirconia (Zr), PEEK CAD (PC) and PEEK press (Pp) groups. Copings were subjected to three dimension (3D) analysis of marginal and internal fit by using a triple scan protocol. Results: The results of this study showed that zirconia group recorded statistically significant $\mathrm{P}=0.02(\mathrm{P}<0.05)$ lower absolute marginal gap mean value $(60 \pm 10 \mu \mathrm{m})$ than PEEK CAD $(69 \pm 0.00 \mu \mathrm{m})$ and PEEK press group $(69 \pm 10 \mu \mathrm{m})$. Among internal fit There was no statistical significant difference between the three groups as $\mathrm{p}=0.36(>0.05)$ but PEEK press showed better internal fit. $(126 \pm 30 \mu \mathrm{m}),(130 \pm 40 \mu \mathrm{m})$ and $(112 \pm 40 \mu \mathrm{m})$ for zirconia, PEEK CAD and PEEK press respectively. Conclusion: Marginal and internal fit for the three groups were within the acceptance range while zirconia copings show significance superior marginal fit than PEEK groups.
\end{abstract}

KEYWORDS: Three-dimensional analysis, Marginal fit, internal fit, PEEK, Zirconia

\section{INTRODUCTION}

The growing interest in tooth-colored high performance polymers can be attributed to improvements in CAD/CAM technology, faster processing and lower cost as well as improved mechanical properties in combination with the advantages of using them in thinner thicknesses as compared to ceramics ${ }^{(1,2)}$. Polyetheretherketone (PEEK) is a synthetic, tooth colored polymeric material that has been used as a biomaterial in orthopedics for many years ${ }^{(3)}$. PEEK has excellent performance such as high mechanical properties, good wear and heat resistance, and excellent chemical resistance ${ }^{(4,5)}$.
Although it's white color, PEEK is not used in monolithic form but only suitable as framework material veneered by resin composite.

In addition the use of Yttria-stabilized tetragonal zirconia polycrystalline (Y-TZP) for fabrication of all ceramic restorations by means of CAD/CAM is common, due to its unique characteristics including excellent biocompatibility, low plaque accumulation and unmatched mechanical properties ${ }^{(6)}$. Moreover zirconia can be used in monolithic form or as a framework veneered by porcelain or resin composite veneering material to improve reparability and absorb the masticatory forces.

\footnotetext{
* MSc Student Crown and Bridge Department, Faculty of Dentistry, AL Azhar University, Cairo, Egypt.

** Professor. Head of Department of Crown and Bridge, Faculty of Dental Medicine, Al-Azhar University in Cairo, Egypt.

*** Lecturer, Department of Crown and Bridge, Faculty of Dental Medicine, AL Azhar University. Cairo, Egypt

- Corresponding author: makky.mohammad@gmail.com
}

DOI: $10.21608 /$ ajdsm.2020.26019.1035 
However, there has not been enough researches on the marginal and internal fit of restorations fabricated with PEEK as compared to zirconia. When the marginal and internal fit is poor, plaque deposition, periodontal destruction, recurrent caries, and ultimately, the failure of the restoration may occur. Therefore, the fit of the dental restoration plays an important role in the long-term success of the prostheses ${ }^{(7)}$.

Measuring the fit with the traditional methods such as sectioning and replica methods, have some problems such as data missing during sectioning and non-accuracy due to contact to dental objects. Triple scan protocol is a new technique which overcomes these problems depending on the revolution on optical (3D) scanning technologies. This method gives three dimensional (3D) view of the space between the FDP and the abutment with unlimited number of points more than (2D) one ${ }^{(8,9)}$.

\section{MATERIALS AND METHODS}

\section{Grouping:}

Twenty one copings were used in this study and equally divided into three groups $(n=7)$ according to the technique of fabrication and the type of material: PEEK CAD (PC): Brecam BioHPP, PEEK press (Pp): BioHPP granulate and zirconia (Zr): Zircon.X.

\section{Preparation of specimen}

Typodont tooth of mandibular first molar was fixed in a plastic mold by self-cure acrylic resin (Acrostone Egypt) with the cervical line of the acrylic tooth was $2 \mathrm{~mm}$ coronal to top surface of the mold. After setting of the self-cure acrylic resin, the tooth was prepared to receive circumferential deep chamfer finish line $1 \mathrm{~mm}$ in thickness and $1.5 \mathrm{~mm}$ occlusal reduction by using laboratory diamond stone with around tip $\left(6^{\circ}\right.$ taper angle and $1 \mathrm{~mm}$ tip diameter) attached to a milling surveyor (BEGO. PARASKOP, Germany).

\section{Digital impression}

For the digital impression, a thin dusting with titanium dioxide powder was applied to facilitate scanning of the prepared tooth. The prepared tooth was scanned with Shera eco scan 7 scanner (Shera Werkstoff Technology, Germany powered by Dental Wings software); restoration parameters were set, including the spacer thickness at $(50 \mu)$ while other parameters were kept according to the software default.

After coping designing; STL file was imported to the milling unit (Shera eco mill 5X, Shera Werkstoff Technology, Germany) software which ordered for milling of fourteen copings (seven for Zircon.x and seven for brecam BioHPP) and seven CAD/milling wax pattern for BioHPP granulate.

\section{Zirconia coping sintering}

The resultant zirconia copings were placed on its occlusal surface on a special glass beads for sintering inside the sintering furnace (Nabertherm, Germany) according to manufacture instructions. The starting temperature was $403{ }^{\circ} \mathrm{C}$ and increased at rate of $20{ }^{\circ} \mathrm{C} / \mathrm{min}$ until $900{ }^{\circ} \mathrm{C}$ and hold for 10 min; then firing was increased at a rate of $10^{\circ} \mathrm{C} / \mathrm{min}$ until 1530 and hold for 2 hours.

\section{Fabrication of pressed Bio HPP copings}

A special device (for 2 press Bredent, Germany) for pressable Bio HPP materials was used, to prevent air pockets inside the coping; a brush is used during the investment process. This ensures that the crown interior is free of bubbles. Then entire mold can then be filled with investment material. After 20 min. setting time, the hot mold is removed from the plastic base using a slight turning motion. In order to enable trouble-free pressing, the muffle must always be positioned straight and at a right angle.

\section{Measurement of the copings fit}

\section{Digitizing of the copings}

The master die and the intaglio of each copings were digitized using 3Shape TRIOS $® 3$ (3Shape 
A/S, Copenhagen K Denmark ,pod and wired type) which is a powder free 3D intra oral scanner with a trueness (accuracy) $(6.9 \pm 0.9 \mu \mathrm{m})$ and precision $(4.5 \pm 0.9 \mu \mathrm{m})^{(10)}$. Three different scans were made according to the triple-scan protocol by Holst et $\mathrm{al}^{(11)}$.

The prepared tooth, the outer and intaglio surfaces of each one of the copings, (a microbrush attached with flowable composite resin to the coping used to allow a free movement of the scanner around the coping) and each coping on the tooth in a clinically correct final position ${ }^{(12)}$. To ensure that every coping correctly seated on the tooth during scanning we hold it by a custom made positioning device.

\section{Data alignment:}

Copings STL files were sent to 3D inspection software (Poly Works Innovmetric 2007, Canada) for fit analysis and 3D color mapping as follow: first, the die STL file (as an object) and the copingdie STL file (as a reference) were registered by manual alignment (prealignment using point pairs) followed by best-fit registration. The same procedures repeated for alignment of coping and coping-die STL files, then coping-die STL file was removed to obtain aligned coping and die data sets in their clinically correct position .The final step of the alignment procedures was to delete the outer surface of the coping and invert the orientation.

\section{Fit assessment}

For the 3D measurements, twenty sections, ten bucco-lingual and ten mesio-distal, were made through aligned data. The distance between the die and the intaglio surface of the crown were measured at 8 standardized points ( 2 on the margins, 2 at $0.75 \mathrm{~mm}$ above the margin, 2 on the mid axial walls and 2 on the occlusal surface), Fig. $1 \& 2$.

Data were represented by mean, standard deviation (SD), median (M), with 95\% Confidence Interval $(95 \% \mathrm{CI})$ values. One way ANOVA test, post hoc tuckey tests and descriptive statistics were used to compare between different materials. The significance level was set to $\mathrm{P} \leq 0.05$. Statistical analysis was performed with $\operatorname{IBM}^{\circledR} *$ SPSS ${ }^{\Theta}{ }^{\Theta}$ Statistics Version 20 at 95\% confidence interval.

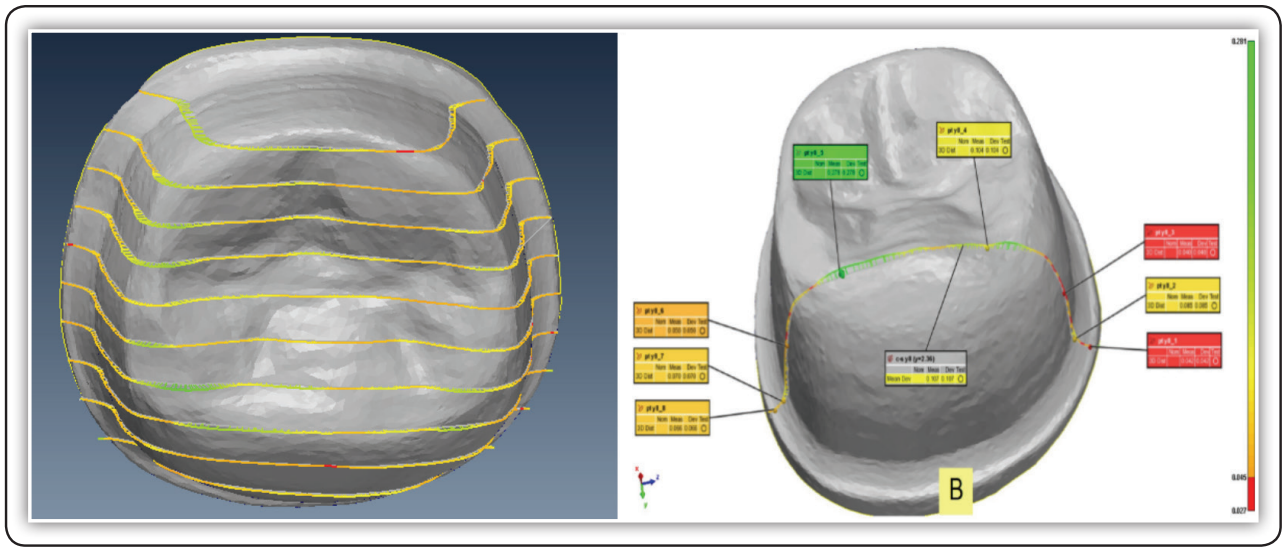

FIG ( $1 \& 2)$ Ten mesio-distal, were made through aligned data. The distance between the die and the intaglio surface of the crown were measured at 8 standardized points ( 2 on the margins, 2 at $0.75 \mathrm{~mm}$ above the margin, 2 on the mid axial walls and 2 on the occlusal surface) 


\section{RESULTS}

\section{Marginal fit}

There was statistical significant difference between $\mathrm{PC}$ and, $\mathrm{Pp}$ and $\mathrm{Zr}$ as $\mathrm{p}=0.004$ and 0.03 respectively while no statistical significant difference between PC and Pp as $p=0.9$, Table (1).

TABLE (1): Descriptive statistics of marginal gap of main groups in $(\mu \mathrm{m})$

\begin{tabular}{|c|c|c|c|c|c|c|}
\hline \multicolumn{7}{|c|}{ Marginal fit } \\
\cline { 1 - 5 } & Mean & SD & Min & Max & CI & P \\
\hline Zr & $60^{\mathrm{a}}$ & 11 & 52 & 74 & 10 & \multirow{2}{*}{$0.02^{\mathrm{A}}$} \\
\cline { 1 - 5 } PC & $69^{\mathrm{b}}$ & 0 & 67 & 78 & 10 & \\
\cline { 1 - 5 } $\mathrm{Pp}$ & $69^{\mathrm{b}}$ & 12 & 60 & 81 & 20 & \\
\hline
\end{tabular}

Large letters for significance, small letters shows difference between the same columns only

\section{Internal fit}

There was no statistical significant difference between the three groups as $p=0.36(>0.05)$ but $\mathrm{Pp}$ showed better internal fit, Table (2).

TABLE (2): Descriptive statistics of internal fit of the main groups in $(\mu \mathrm{m})$

\begin{tabular}{|c|c|c|c|c|c|c|}
\hline \multicolumn{7}{|c|}{ Internal fit } \\
\cline { 1 - 6 } & Mean & SD & Min & Max & CI (95\%) & P \\
\cline { 1 - 6 } Zr & 127 & 34 & 93 & 179 & 20 & \\
PC & 129 & 40 & 98 & 223 & 30 & \multirow{2}{*}{0.36} \\
\hline Pp & 111 & 41 & 62 & 171 & 30 & \\
\hline
\end{tabular}

For average of axial mid points there was statistically significant difference between Pp, PC and $\mathrm{Zr}$ as $\mathrm{p}=0.00$. There was statistically significant difference between $\mathrm{Pp}$ and $\mathrm{PC}$, as $\mathrm{p}=0.008$ and between $\mathrm{PP}$, and $\mathrm{Zr} 0.006$ respectively while there was no statistically significant difference between $\mathrm{PC}$ and $\mathrm{Zr}$ as $\mathrm{p}=0.06$.

For average of axial Cervical points there was no statistically significant difference between PP, PC and $\mathrm{Zr}$ groups as $\mathrm{p}=0.3$ There was no statistically significant difference between $\mathrm{PP}, \mathrm{PC}$ and $\mathrm{Zr}$ groups at mesiodistal and buccolingual points as $\mathrm{p}$ $=0.37$ and 0.16 respectively. For average of axial occlusal points there was no statistically significant difference between $\mathrm{PP}, \mathrm{PC}$ and $\mathrm{Zr}$ groups as $\mathrm{p}=$ 0.26 , Table (3).

TABLE (3): Show internal fit of the main groups at each measuring point $(\mu \mathrm{m})$.

\begin{tabular}{|c|c|c|c|c|c|c|}
\hline \multicolumn{7}{|c|}{ Internal fit at each point } \\
\hline & & & $\mathrm{Pp}$ & $\mathrm{PC}$ & $\mathrm{Zr}$ & $\mathrm{P}$ \\
\hline \multirow{4}{*}{$\begin{array}{l}\text { Axial } \\
\text { points }\end{array}$} & \multirow{2}{*}{$\begin{array}{l}\text { Mid } \\
\text { points }\end{array}$} & MEAN & $72^{\mathrm{a}}$ & $111^{\mathrm{b}}$ & $102^{\mathrm{b}}$ & \multirow{2}{*}{$0.00^{A}$} \\
\hline & & SD & 130 & 140 & 100 & \\
\hline & \multirow{2}{*}{$\begin{array}{l}\text { Cervical } \\
\text { points }\end{array}$} & MEAN & 119 & 120 & 140 & \multirow{2}{*}{0.35} \\
\hline & & SD & 10 & 11 & 23 & \\
\hline \multirow{2}{*}{\multicolumn{2}{|c|}{$\begin{array}{l}\text { Occlusal points } \\
\text { SD }\end{array}$}} & MEAN & 98 & 127 & 106 & \multirow{2}{*}{0.26} \\
\hline & & 82 & 94 & 79 & & \\
\hline
\end{tabular}

Large letters for significance, small letters shows difference between the same columns only.

\section{DISCUSSION}

Accuracy of fit of fixed dental restorations is usually defined by measuring parameters like marginal and internal gap. Sufficient marginal adaptation reduces the risk of secondary caries, cement dissolutions and gum inflammations. Moreover the internal fit has a significant influence on the mechanical properties of the restorations in terms of fracture resistance and retention. The final fit of the restoration sums up the errors that occur both in the manufacturing process along with the clinical setting ${ }^{(13)}$.

Ayad ${ }^{(14)}$ found significant differences among the die material used for the shoulder margin design with greater marginal opening than light chamfer and deep chamfer margin designs for both die materials. So in this study a deep chamfer finish line was used that is compatible with non-metallic restorations and decrease stress and its fracture liability. 
CAD/CAM technology was chosen due to its ability to control thickness and anatomy of restorations during the fabrication process. It also allowed the standardization of the internal fit of the restoration as well as the mechanical properties of the restorative materials. Many potentially confounding operator variables were avoided such as dental laboratory technicians' skills and procedures involved in the fabrication process ${ }^{(15)}$.

For standardization for all samples, single typodont prepared die scanned extraorally by one operator to fabricate CAD design .we directly scan the prepared tooth for coping milling without making a die replica as the measuring technique didn't require direct contact between tooth and the coping ${ }^{(16)}$.

For pressed group we made a $\mathrm{CAD} /$ milling of wax patterns with the same parameters of CAD groups milling to ensure standardization of cement gap and coping thickness ${ }^{(17)}$. Also wax patterns of all pressed copings invested together in same mold while finishing and polishing is done by one operator. During sintering of zirconia to avoid margin distortion copings put in upside down position on its occlusal surface on glass bead.

This study, not only tested the new technology available in dentistry, but also utilized similar industrial methodology in obtaining the measurements of the digital specimens. The 3D data were obtained by digitizing the physical specimens. The digital models could be moved, rotated, sectioned and measured as many times and in any way as desired. This method has the potential of providing a vast amount of information that was not possible before.

Rajshekar et al ${ }^{(18)}$ stated that intra-oral 3D scanner a reliable and valid method for measuring the key features of virtual 3D dental casts. This technique has excellent potential for recording and accurately measuring dental features for forensic purposes including bite-mark analysis, and for research purposes. Agnes et al ${ }^{(19)}$ estimate dental wear using a digital intra-oral optical scanner and also stated intra oral scanner is a potential resource to allow reliable comparison of measurements over time.

Park et al ${ }^{(11)}$ use intra oral scanners for in vivo assessment of internal and marginal fit of fixed dental prostheses. Intraoral scanner (IOS) allow scanning of outer and internal surface of the restoration in one shot unlike of industrial 3D scanners or coordinate measuring machine (CMM) which scan the surfaces independently then ingrate them by software ${ }^{(12)}$.Software image integration is done by best fit alignment which sometimes meet difficulties as in case of round smooth surfaces We can also use IOS to clinically check the marginal and internal fit of the restoration.

Where high accuracy measurements are needed we need to use a highly precise measuring noncontact 3D scanner so we used in this study a 3Shape TRIOS@3 intraoral scanner which has a trueness $(6.9 \pm 0.9 \mu \mathrm{m})$ and precision $(4.5 \pm 0.9 \mu \mathrm{m})$ ${ }^{(10)}$.Unlike most other scanners, 3Shape TRIOS ${ }^{\circledR}$ is a no-spray solution, because spraying is technique demanding, can affect scan accuracy, and no need to hold the scanner at a specific distance or angle for focus. To avoid surface affection of the prepared acrylic tooth due to repeated coping placement during scanning, we scan firstly the tooth which later used for data alignment for all samples.

The results of this study showed that zirconia group recorded statistically significant $\mathrm{P}=0.02$ $(\mathrm{P}<0.05)$ lower absolute marginal gap mean value $(60 \pm 10 \mu \mathrm{m})$ than PEEK CAD $(69 \pm 0.00 \mu \mathrm{m})$ and PEEK press group $(69 \pm 10 \mu \mathrm{m})$. Despite the statistical difference between zirconia and PEEK and the remaining three groups for the marginal fit, the clinical difference might not be of great importance. Unlike results with a study by Young Park et al (20) which revealed higher non-significant marginal discrepancy values of Zirconia crowns (77.06 \pm 32.14 $\mu \mathrm{m})$ than those of poly etherketoneketone (PEKK) which is a member of poly arylketone family $(66.83 \pm 22.31 \mu \mathrm{m})$. 
Also same results of study by Park et al ${ }^{(9)}$ study stated that marginal and internal fit of both PEKK and zirconia copings were within the clinically acceptable range. However, the PEKK presented better fitness compared with the zirconia. In comparison with PEEK materials, PEKK materials present stronger solidification of the glass and polymer chains due to the high ratio of the keto group; hence, it has a higher melting temperature and greater compressive strength ${ }^{(21)}$.

According to Guess et al., ${ }^{(22)} 100 \mu \mathrm{m}$ is the clinically acceptable marginal gap for ceramics, while McLean and von Frauenhofer ${ }^{23)}$ reported a gap of less than $120 \mu \mathrm{m}$. Another previous study reported that $100-200 \mu \mathrm{m}$ is the clinically acceptable range for long-term preserved dental prostheses $^{(24)}$.

Among internal fit There was no statistical significant difference between the three groups as $\mathrm{p}=0.36(>0.05)$ but PEEK press showed better internal fit. $(126 \pm 30 \mu \mathrm{m}),(130 \pm 40 \mu \mathrm{m})$ and $(112 \pm 40 \mu \mathrm{m})$ for zirconia, PEEK CAD and PEEK press respectively. These results coincide with other studies stated that pressed FDPs show superior fit than CAD ones ${ }^{(20,25-27)}$. It was attributed to the type of manufacturing of these two systems; the distortion of the ceramic coping was thought to be less in PEEK press compared with CAD/CAM, as the pressed technique involves a less complicated process, using a more manual than computer program computation, and is process dependent.

The present study showed that in terms of the occlusal region, (PP 98 $\pm 82 \mu \mathrm{m}),($ PC $127 \pm 94$ $\mu \mathrm{m})$ and $(\mathrm{Zr} \mathrm{106} \pm 79 \mu \mathrm{m})$ exceeded the axial mid points (PP $72 \pm 130 \mu \mathrm{m}),(\mathrm{PC} 111 \pm 140 \mu \mathrm{m})$ and (Zr $102 \pm 100 \mu \mathrm{m})$ of internal adaptation. Also cervical points (PP $119 \pm 110 \mu \mathrm{m}),(\mathrm{PC} 120 \pm 11 \mu \mathrm{m})$ and $(\mathrm{Zr}$ $140 \pm 23 \mu \mathrm{m})$ showed values greater than axial mid points. These results coincide with other studies ${ }^{(20}$, ${ }^{28)}$. It could be partially explained by limitations in the scanner resolution, which may produce slightly rounded edges. A phenomenon called "overshoot" that occurs during the scanning process could be transformed by the software in an area with internal imperfections that can result in adaptation interferences in both marginal and occlusal $\operatorname{areas}^{(29,30)}$.

Also because the axial line angles the occlusal surface of the molar region was not an exact reproduction due to the bur diameter limitation at the undercut site. ${ }^{(20,31)}$ However, the measurement value of the occlusal region in the present study was clinically acceptable according to previous reports (32,33).

Because of the high variation of fit within any particular crown system, the mean values of various measurement locations can demonstrate a large local discrepancy and may result in an increase in the overall standard deviations SDs. The mean values of the present study were accompanied by large SDs. The fit of a particular location might further be influenced be influenced by the asymmetric anatomical shape of the copings ${ }^{(34,35)}$.

\section{CONCLUSION}

Within the limitation of this study, the following conclusions were drawn:

1. Marginal and internal fit for the three groups were within the acceptance range.

2. Zirconia copings showed significance superior marginal fit than PEEK groups regardless of its technique (CAD/CAM or press technique).

\section{REFERENCES}

1. Wimmer T, Huffmann AMS, Eichberger M, Schmidlin PR, Stawarczyk BJCOI. Two-body wear rate of PEEK, CAD/CAM resin composite and PMMA: Effect of specimen geometries, antagonist materials and test set-up configuration. J Dental Materials. 2016;32(6):e127-e36.

2. Stawarczyk B, Ender A, Trottmann A, Özcan M, Fischer J, Hämmerle CHJCoi. Load-bearing capacity of CAD/ CAM milled polymeric three-unit fixed dental prostheses: effect of aging regimens. J Clinical oral investigations 2012;16(6):1669-77. 
3. Pokorný D, Fulin P, Slouf M, Jahoda D, Landor I, Sosna AJAcoetC. Polyetheretherketone (PEEK). Part II: Application in clinical practice. Acta chirurgiae orthopaedicae et traumatologiae Cechoslovaca. 2010;77(6):470-8.

4. Yamamoto Y, Takashima TJW. Friction and wear of water lubricated PEEK and PPS sliding contacts. J Wear 2002;253(7-8):820-6.

5. Zhang G, Liao H, Cherigui M, Davim JP, Coddet CJTJopd. Effect of crystalline structure on the hardness and interfacial adherence of flame sprayed poly (ether-etherketone) coatings. The Journal of prosthetic dentistry 2007;43(3):1077-82.

6. Bachhav VC, Aras MAJQi. Zirconia-based fixed partial dentures: A clinical review. Quintessence international 2011;42(2).

7. Song T-J, Kwon T-K, Yang J-H, Han J-S, Lee J-B, Kim $\mathrm{S}-\mathrm{H}$, et al. Marginal fit of anterior 3-unit fixed partial zirconia restorations using different CAD/CAM systems. J Adv Prosthodont. 2013;5(3):219-25.

8. Dahl BE, Dahl JE, Rønold HJJEjoos. Internal fit of threeunit fixed dental prostheses produced by computer-aided design/computer-aided manufacturing and the lost-wax metal casting technique assessed using the triple-scan protocol. European journal of oral sciences 2018;126(1): 66-73.

9. Bae S-Y, Park J-Y, Jeong I-D, Kim H-Y, Kim J-H, Kim W-CJJopr. Three-dimensional analysis of marginal and internal fit of copings fabricated with polyetherketoneketone (PEKK) and zirconia. Journal of prosthodontic research 2017;61(2):106-12.

10. Hack SBMPaGD. Evaluation of the Accuracy of Six Intraoral Scanning Devices: An in-vitro Investigation. American Dental Association. 2015;10(4):5.

11. Holst S, Karl M, Wichmann M, Matta R-ETJQI. A new triple-scan protocol for 3D fit assessment of dental restorations. J Quintessence International 2011;42(8).

12. Park J-M, Hämmerle CH, Benic GIJTJopd. Digital technique for in vivo assessment of internal and marginal fit of fixed dental prostheses. The Journal of prosthetic dentistry 2017;118(4):452-4.

13. Dauti R, Cvikl B, Lilaj B, Heimel P, Moritz A, Schedle AJJopr. Micro-CT evaluation of marginal and internal fit of cemented polymer infiltrated ceramic network material crowns manufactured after conventional and digital impressions. Journal of prosthodontic research 2019;63(1):40-6
14. Ayad MFJJCDP. Effect of the crown preparation margin and die type on the marginal accuracy of fiber-reinforced composite crowns. Contemp Dent Pract 2008;9(2):9-16.

15. Beuer F, Schweiger J, Edelhoff DJBdj. Digital dentistry: an overview of recent developments for CAD/CAM generated restorations. British dental journal 2008;204(9):505.

16. Ferrini F, Sannino G, Chiola C, Capparè P, Gastaldi G, Gherlone E. Influence of Intra-Oral Scanner (I.O.S.) on The Marginal Accuracy of CAD/CAM Single Crowns. International Journal of Environmental Research and Public Health. 2019;16:544.

17. Ghodsi S, Alikhasi M, Soltani NJEjod. Marginal Discrepancy of Single Implant-Supported Metal Copings Fabricated by Various CAD/CAM and Conventional Techniques Using Different Materials. European journal of dentistry 2019.

18. Rajshekar M, Julian R, Williams A-M, Tennant M, Forrest $\mathrm{A}$, Walsh $\mathrm{LJ}$, et al. The reliability and validity of measurements of human dental casts made by an intra-oral 3D scanner, with conventional hand-held digital callipers as the comparison measure. J Forensic science international. 2017;278:198-204.

19. Meireles AB, Vieira AW, Corpas L, Vandenberghe B, Bastos FS, Lambrechts P, et al. Dental wear estimation using a digital intra-oral optical scanner and an automated 3D computer vision method. J Computer methods in biomechanics biomedical engineering. 2016;19(5):507-14.

20. Park J-Y, Bae S-Y, Lee J-J, Kim J-H, Kim H-Y, Kim W-C. Evaluation of the marginal and internal gaps of three different dental prostheses: comparison of the silicone replica technique and three-dimensional superimposition analysis. J Adv Prosthodont. 2017;9(3):159-69.

21. Fuhrmann G, Steiner M, Freitag-Wolf S, Kern M. Resin bonding to three types of polyaryletherketones (PAEKs) durability and influence of surface conditioning. Dental Materials. 2014;30(3):357-63\%@ 0109-5641.

22. Guess PC, Vagkopoulou T, Zhang Y, Wolkewitz M, Strub JRJDm. Marginal and internal fit of heat pressed versus CAD/CAM fabricated all-ceramic onlays after exposure to thermo-mechanical fatigue. J Dental materials. 2014;42(2):199-209.

23. McLean JJBdj. The estimation of cement film thickness by an in vivo technique. J Br dent j. 1971;131:107-11.

24. Bindl A, Mörmann WJJoor. Marginal and internal fit of all-ceramic CAD/CAM crown-copings on chamfer preparations. J Journal of oral rehabilitation. 2005;32(6):441-7. 
25. Stappert CF, Chitmongkolsuk S, Silva NR, Att W, Strub JRJDm. Effect of mouth-motion fatigue and thermal cycling on the marginal accuracy of partial coverage restorations made of various dental materials. J Dental materials. 2008;24(9):1248-57.

26. Baig MR, Tan KB-C, Nicholls JIJTJopd. Evaluation of the marginal fit of a zirconia ceramic computer-aided machined (CAM) crown system. J The Journal of prosthetic dentistry. 2010;104(4):216-27.

27. Anadioti E. Internal and marginal fit of pressed and cad lithium disilicate crowns made from digital and conventional impressions. University of Iowa. 2013.

28. Bayramoğlu E, Özkan YK, Yildiz CJTJopd. Comparison of marginal and internal fit of press-on-metal and conventional ceramic systems for three-and four-unit implantsupported partial fixed dental prostheses: An in vitro study. J The Journal of prosthetic dentistry. 2015;114(1):52-8.

29. Willer J, Rossbach A, Weber H-PJTJopd. Computerassisted milling of dental restorations using a new CAD/ CAM data acquisition system. The Journal of prosthetic dentistry. 1998;80(3):346-53.

30. Luthardt R, Weber A, Rudolph H, Schöne C, Quaas S, Walter MJIjocd. Design and production of dental prosthetic restorations: basic research on dental CAD/CAM tech- nology. J International journal of computerized dentistry. 2002;5(2-3):165-76.

31. Abduo J, Lyons K, Bennamoun MJIjod. Trends in computer-aided manufacturing in prosthodontics: a review of the available streams. J International journal of dentistry. 2014;2014.

32. Guess PC, Vagkopoulou T, Zhang Y, Wolkewitz M, Strub JR. Marginal and internal fit of heat pressed versus CAD/CAM fabricated all-ceramic onlays after exposure to thermo-mechanical fatigue. Journal of dentistry. 2014;42(2):199-209.

33. Örtorp A, Jönsson D, Mouhsen A, von Steyern PVJDM. The fit of cobalt-chromium three-unit fixed dental prostheses fabricated with four different techniques: A comparative in vitro study. J Dental Materials. 2011;27(4):356-63.

34. Riccitiello F, Amato M, Leone R, Spagnuolo G, Sorrentino RJTodj. In vitro evaluation of the marginal fit and internal adaptation of zirconia and lithium disilicate single crowns: micro-CT comparison between different manufacturing procedures. J The open dentistry journal 2018;12:160.

35. Baig MR, Tan KB-C, Nicholls JJJTJopd. Evaluation of the marginal fit of a zirconia ceramic computer-aided machined (CAM) crown system. J The Journal of prosthetic dentistry 2010;104(4):216-27. 